\title{
EPW: A program for calculating the electron-phonon coupling using maximally localized Wannier functions
}

\author{
Jesse Noffsinger $*$ Brad D. Malone, Cheol-Hwan Park, Steven G. Louie, and Marvin L. Cohen \\ Department of Physics, University of California, Berkeley, California 94720, USA. and \\ Materials Sciences Division, Lawrence Berkeley National Laboratory, Berkeley, California 94720, USA.
}

\author{
Feliciano Giustino \\ Department of Materials, University of Oxford, Parks Road, Oxford, OX1 3PH, UK.
}

(Dated: May 26, 2010)

\begin{abstract}
EPW (Electron-Phonon coupling using Wannier functions) is a program written in FORTRAN90 for calculating the electron-phonon coupling in periodic systems using density-functional perturbation theory and maximally-localized Wannier functions. EPW can calculate electron-phonon interaction self-energies, electron-phonon spectral functions, and total as well as mode-resolved electron-phonon coupling strengths. The calculation of the electron-phonon coupling requires a very accurate sampling of electron-phonon scattering processes throughout the Brillouin zone, hence reliable calculations can be prohibitively time-consuming. EPW combines the Kohn-Sham electronic eigenstates and the vibrational eigenmodes provided by the Quantum-ESPRESSO package [1] with the maximally localized Wannier functions provided by the wannier90 package [2] in order to generate electron-phonon matrix elements on arbitrarily dense Brillouin zone grids using a generalized Fourier interpolation. This feature of EPW leads to fast and accurate calculations of the electron-phonon coupling, and enables the study of the electron-phonon coupling in large and complex systems.
\end{abstract}

PACS numbers: 63.20.kd, 63.20.kk, 71.15.-m, 74.25.Kc, 74.70.-b

\section{PROGRAM SUMMARY}

Program Title: EPW

Journal Reference:

Catalogue identifier:

Licensing provisions: GNU Public License

Programming language: Fortran 90

Computer: any architecture with a Fortran 90 compiler

$R A M$ : heavily system dependent, as small as a few MB

Number of processors used: optimized for 1 to 64 processors

Classification: 7

External routines/libraries: BLAS, LAPACK, MPI, FFTW

Subprograms used: wannier90

Nature of problem: The calculation of the electron-phonon coupling from first principles requires a very accurate sampling of electron-phonon scattering processes throughout the Brillouin zone; hence reliable calculations can be prohibitively timeconsuming.

Solution method: EPW makes use of a real-space formulation and combines the Kohn-Sham electronic eigenstates and the vibrational eigenmodes provided by the Quantum-ESPRESSO package with the maximally localized Wannier functions provided by the wannier90 package in order to generate electron-phonon matrix elements on arbitrarily dense Brillouin zone grids using a generalized Fourier interpolation.

Running time: single processor examples typically take 5-10 minutes

References: Giustino, F., Cohen, M. L., and Louie, S. G., Physical Review B 76 (2007) 165108.

\section{INTRODUCTION}

The electron-phonon interaction plays a crucial role in the electron and lattice dynamics of condensed matter systems. For example, phenomena such as the electrical resistivity [3] and conventional superconductivity [4] are a direct consequence of the interaction between electrons and lattice vibrations. The electron-phonon interaction also plays an important role in the thermoelectric effect [5]. Other fundamental physical phenomena such as the Kohn effect [6] and the Peierls [7] distortions are also direct consequences of the electron-phonon interaction. The electron-phonon interaction is also responsible for the broadening of the spectral lines in angle-resolved photoemission spectroscopy [8] and in vibrational spectroscopies [9], as well as for the temperature dependence of the band gaps in 
semiconductors [10].

The calculation of the electron-phonon coupling from first-principles is challenging because of the necessity of evaluating Brillouin zone integrals with high accuracy. Such calculation requires the evaluation of matrix elements between electronic states connected by phonon wavevectors [11]. Well-established software packages are available for computing electronic states and eigenvalues through density-functional theory [1, 12, 13], as well as phonon frequencies and eigenmodes through density-functional perturbation theory [14]. However large numbers of matrix elements may be necessary to achieve numerical convergence of the Brillouin zone intergrals over these matrix elements.

For example, in order to compute the electronic lifetimes associated with the electron-phonon interaction it is necessary to evaluate a Brillouin zone integral over all the possible phonon wavevectors (thousands to millions). Since lattice-dynamical calculations for each phonon wavevector are at least as expensive as self-consistent total energy minimizations, achieving numerical convergence in the Brillouin zone integrals over the phonon wavevectors by bruteforce calculations may become a prohibitive computational task. EPW exploits the real-space localization of Wannier functions to generate large numbers of first-principles electron-phonon matrix elements through a generalized Fourier interpolation. EPW therefore enables affordable, accurate, and extremely efficient calculations of the electron-phonon coupling [15]. The use of maximally localized Wannier functions (MLWFs) 16, 17] to calculate Brillouin zone integrals with high accuracy has been the object of a number of other studies [18 30].

In this communication we outline the functionalities of EPW and the details of the technical release (Sec. II), we review the individual sections of the code (Sec. III), and we describe its parallel structure (Sec. VI). We then illustrate the capabilities of EPW through example calculations (Sec. V). We finally discuss some future directions for additional development (Sec. VI). Some technical details are given in the Appendix (Sec. VII).

\section{FUNCTIONALITIES AND TECHNICAL RELEASE}

EPW is a program written in FORTRAN90 which calculates the electron-phonon coupling from first principles using maximally localized Wannier functions. EPW uses information provided by Quantum-ESPRESSO [1] and wannier9o [2], and runs as a post-processing tool. Electrons are described using density functional theory (DFT) [12, 13] with planewaves and pseudopotentials, either separable norm-conserving 31 34] or Vanderbilt ultrasoft [35]. Lattice dynamical properties are calculated within density functional perturbation theory (DFPT) 14]. The theoretical background and methodology are thoroughly described in Ref. [15]. Examples of quantities [36] which can be computed using EPW include:

- the total electron-phonon coupling strength $\lambda$,

- the phonon self-energy associated with the electron-phonon interaction within the Migdal approximation,

- the electron self-energy associated with the electron-phonon interaction within the Migdal approximation,

- the Eliashberg electron-phonon spectral function $\alpha^{2} F$,

- the transport electron-phonon spectral function $\alpha^{2} F_{\mathrm{T}}$.

EPW is freely available under the GNU General Public License (GPL). The current version is developed and maintained using Subversion and is accessible to prospective developers and end-users at the website epw.org.uk. EPW employs the freely available FFTW, BLAS, LAPACK libraries in conjunction with several subroutines distributed within the Quantum-ESPRESSO package. Several subroutines in EPW are based upon modified Quantum-ESPRESSO subroutines as permitted under the GPL. The parallelization is achieved through the MPI library specification for message passing. The current version of EPW, v2.3, includes approximately 9000 independent lines of FORTRAN90 code. In addition to the source code, several complete example calculations are provided with the EPW distribution.

The inputs to EPW are as follows:

- Phonon dynamical matrices for the wavevectors of a uniform Brillouin-zone grid centered at $\Gamma$ (prefix.dyn files from Quantum-ESPRESSO). Only wavevectors within the irreducible wedge of the Brillouin zone are required.

- The derivatives of the self-consistent potential with respect to the phonon perturbations, for the same wavevectors as above (prefix.dvscf files from Quantum-ESPRESSO).

- The electron eigenfunctions and eigenvalues for the wavevectors of a uniform Brillouin-zone grid centered at $\Gamma$ (prefix.wfc or prefix.save/ files from Quantum-ESPRESSO). 
- Norm-conserving pseudopotentials [32] or Vanderbilt ultrasoft pseudopotentials [35],

- A plain text input file specifying the runtime parameters.

The electronic wavefunctions are calculated on a uniform grid using Quantum-ESPRESSO. Dynamical matrices and the derivatives of the self-consistent potential are also computed within Quantum-ESPRESSO for phonons in the irreducible wedge of the Brillouin zone. When choosing initial electron and phonon grids, it is necessary that the Brillouin zone grid for phonons be comensurate with the Brillouin zone grid for electrons in order to map the wavefunctions $\psi_{m \mathbf{k}+\mathbf{q}}(\mathbf{r})$ onto $\psi_{m \mathbf{k}^{\prime}+\mathbf{G}}(\mathbf{r})$, with $\mathbf{G}$ a reciprocal lattice vector. For example, if the calculation is performed using a Brillouin zone grid of size $6 \times 6 \times 6$ for the phonons, then the natural choices for the electronic Brillouin zone grid are either $6 \times 6 \times 6$ or $12 \times 12 \times 12$. This does not represent a computational bottleneck as phonon calculations are considerably more time-consuming than non-selfconsistent electronic calculations.

\section{COMPUTATIONAL METHODOLOGY}

\section{A. Physical quantities (selfen_phon, selfen_elec, a2f)}

In this section we describe some of the physical quantities which can be calculated using EPW. The imaginary part of the phonon self-energy within the Migdal approximation [36, 37] is calculated as:

$$
\Pi_{\mathbf{q} \nu}^{\prime \prime}=\operatorname{Im} \sum_{m n, \mathbf{k}} w_{\mathbf{k}}\left|g_{m n}^{\nu}(\mathbf{k}, \mathbf{q})\right|^{2} \frac{f\left(\epsilon_{n \mathbf{k}}\right)-f\left(\epsilon_{m \mathbf{k}+\mathbf{q}}\right)}{\epsilon_{n \mathbf{k}}-\epsilon_{m \mathbf{k}+\mathbf{q}}-\omega_{\mathbf{q} \nu}+i \eta} .
$$

In Eq. (11) the electron-phonon matrix elements are given by

$$
g_{m n}^{\nu}(\mathbf{k}, \mathbf{q})=\left\langle\psi_{m \mathbf{k}+\mathbf{q}}\left|\partial_{\mathbf{q} \nu} V\right| \psi_{n \mathbf{k}}\right\rangle,
$$

with $\psi_{n \mathbf{k}}$ the electronic wavefunction for band $m$, wavevector $\mathbf{k}$, and eigenvalue $\epsilon_{n \mathbf{k}}, \partial_{\mathbf{q} \nu} V$ the derivative of the self-consistent potential associated with a phonon of wavevector $\mathbf{q}$, branch index $\nu$, and frequency $\omega_{\mathbf{q} \nu}$. The factors $f\left(\epsilon_{n \mathbf{k}}\right), f\left(\epsilon_{m \mathbf{k}+\mathbf{q}}\right)$ in Eq. (11) are the Fermi occupations, and $w_{\mathbf{k}}$ are the weights of the $\mathbf{k}$-points normalized to 2 in order to account for the spin degeneracy in spin-unpolarized calculations. A very common approximation to Eq. (10) consists of neglecting the phonon frequencies $\omega_{\mathbf{q} \nu}$ and taking the limit of small broadening $\eta$. The final expression is positive definite and is often referred to as the "double-delta function" approximation [36]. This approximation is no longer necessary when using EPW. We note that the imaginary part of the phonon self-energy in Eq. (1) also corresponds to the phonon half-width at half-maximum $\gamma_{\mathbf{q} \nu}$.

The electron-phonon coupling strength associated with a specific phonon mode and wavevector $\lambda_{\mathbf{q} \nu}$ is given by

$$
\lambda_{\mathbf{q} \nu}=\frac{1}{N_{\mathrm{F}} \omega_{\mathbf{q} \nu}} \sum_{m n, \mathbf{k}} w_{\mathbf{k}}\left|g_{m n}^{\nu}(\mathbf{k}, \mathbf{q})\right|^{2} \delta\left(\epsilon_{n \mathbf{k}}\right) \delta\left(\epsilon_{m \mathbf{k}+\mathbf{q}}\right),
$$

with $\delta$ being the Dirac delta function. In the double-delta function approximation the coupling strength $\lambda_{\mathbf{q} \nu}$ can be related to the imaginary part of the phonon self-energy $\Pi_{\mathbf{q} \nu}^{\prime \prime}$ as follows:

$$
\lambda_{\mathbf{q} \nu}=\frac{1}{\pi N_{\mathrm{F}}} \frac{\Pi_{\mathbf{q} \nu}^{\prime \prime}}{\omega_{\mathbf{q} \nu}^{2}}
$$

The total electron-phonon coupling $\lambda$ is calculated as the Brillouin-zone average of the mode-resolved coupling strengths $\lambda_{\mathbf{q} \nu}$ :

$$
\lambda=\sum_{\mathbf{q} \nu} w_{\mathbf{q}} \lambda_{\mathbf{q} \nu}
$$

In Eq. (5) the $w_{\mathbf{q}}$ are the Brillouin zone weights associated with the phonon wavevectors $\mathbf{q}$, normalized to 1 in the Brillouin zone. The Eliashberg spectral function $\alpha^{2} F$ can be calculated in terms of the mode-resolved coupling strengths $\lambda_{\mathbf{q} \nu}$ and the phonon frequencies using:

$$
\alpha^{2} F(\omega)=\frac{1}{2} \sum_{\mathbf{q} \nu} w_{\mathbf{q}} \omega_{\mathbf{q} \nu} \lambda_{\mathbf{q} \nu} \delta\left(\omega-\omega_{\mathbf{q} \nu}\right) .
$$


The transport spectral function $\alpha^{2} F_{\mathrm{T}}$ is obtained from the Eliashberg spectral function $\alpha^{2} F$ by replacing $\lambda_{\mathbf{q} \nu}$ with $\lambda_{\mathrm{T}, \mathbf{q} \nu}$ :

$$
\begin{gathered}
\alpha^{2} F_{\mathrm{T}}(\omega)=\frac{1}{2} \sum_{\mathbf{q} \nu} w_{\mathbf{q}} \omega_{\mathbf{q} \nu} \lambda_{\mathrm{T}, \mathbf{q} \nu} \delta\left(\omega-\omega_{\mathbf{q} \nu}\right) \\
\lambda_{\mathrm{T}, \mathbf{q} \nu}=\frac{1}{N_{\mathrm{F}} \omega_{\mathbf{q} \nu}} \sum_{m n, \mathbf{k}} w_{\mathbf{k}}\left|g_{m n}^{\nu}(\mathbf{k}, \mathbf{q})\right|^{2} \delta\left(\epsilon_{n \mathbf{k}}\right) \delta\left(\epsilon_{m \mathbf{k}+\mathbf{q}}\right)\left(1-\frac{\mathbf{v}_{n \mathbf{k}} \cdot \mathbf{v}_{m \mathbf{k}+\mathbf{q}}}{\left|\mathbf{v}_{n \mathbf{k}}\right|^{2}}\right),
\end{gathered}
$$

with $\mathbf{v}_{n \mathbf{k}}=\nabla_{\mathbf{k}} \epsilon_{n \mathbf{k}}$ the electron velocity.

The real and imaginary parts of the electron self-energy $\Sigma_{n \mathbf{k}}=\Sigma_{n \mathbf{k}}^{\prime}+i \Sigma_{n \mathbf{k}}^{\prime \prime}$ can be calculated as

$$
\Sigma_{n \mathbf{k}}=2 \sum_{\mathbf{q} \nu} w_{\mathbf{q}}\left|g_{m n}^{\nu}(\mathbf{k}, \mathbf{q})\right|^{2}\left[\frac{n\left(\omega_{\mathbf{q} \nu}\right)+f\left(\epsilon_{m \mathbf{k}+\mathbf{q}}\right)}{\epsilon_{n \mathbf{k}}-\epsilon_{m \mathbf{k}+\mathbf{q}}+\omega_{\mathbf{q} \nu}-i \eta}+\frac{n\left(\omega_{\mathbf{q} \nu}\right)+1-f\left(\epsilon_{m \mathbf{k}+\mathbf{q}}\right)}{\epsilon_{n \mathbf{k}}-\epsilon_{m \mathbf{k}+\mathbf{q}}-\omega_{\mathbf{q} \nu}+i \eta}\right],
$$

with $n\left(\omega_{\mathbf{q} \nu}\right)$ the Bose occupation factors.

\section{B. Calculation of the matrix elements on the coarse Brillouin zone grid (elphon_shuffle_wrap)}

The key task of EPW is to calculate from first-principles electron-phonon matrix elements for a large number of electron and phonon wavevectors with a modest computational effort. The initial step of this process is to determine the electron-phonon matrix elements on coarse grids of electron and phonon wavevectors using standard DFT and DFPT methods [38]. The result of this step is a set of matrix elements given by Eq. (2). The wavefunctions and the phonon perturbation potentials are read into EPW, then the matrix elements on the coarse grid are calculated within EPW.

The phonon dynamical matrices and the linear variations of the self-consistent potential are read from file only for wavevectors in the irreducible wedge of the Brillouin zone. The dynamical matrices and the potentials variations for all the remaining points of the uniform grid are generated using crystal symmetry operations. This strategy is advantageous since the computation of the phonon dynamical matrices and potential variations is generally the most time-consuming part of an electron-phonon calculation. For example, a system with cubic symmetry requiring a coarse mesh of $8 \times 8 \times 8$ phonon wavevectors needs only have phonons calculated at 29 irreducible points; hence the reduction in computational time is a factor of $512 / 29$.

The electron-phonon matrix elements and the dynamical matrices thus calculated at each point of the coarse Brillouin-zone grid can be written to disk through an input file option (prefix.epb files). Subsequent executions of EPW can forgo the recalculation of the matrix elements and dynamical matrices by reading these data from disk.

\section{Interface with wannier90 (pw2wan90epw)}

wannier90 generates maximally localized Wannier functions by minimizing the spread of the Berry-phase position operator through a unitary transform [16]. Details on how to run wannier90 can be found in the wannier90 documentation [2].

In standalone mode wannier90 reads three files generated from a first-principles calculation (prefix.mmn, prefix.amn, prefix.eig), a file which defines the starting guess for determining MLWFs and the crystal structure (prefix.nnkp), and a runtime input file (prefix.win). The execution of wannier90 therefore requires the user to run multiple programs and handle the files to be passed between wannier90 and Quantum-ESPRESSO. In order to simplify the calculation of the electron-phonon coupling EPW calls wannier90 as a library. EPW therefore requires only the wavefunction files from Quantum-ESPRESSO and a runtime input file in order to determine MLWFs using wannier90. The quantites required for running wannier90 are either calculated within EPW (for instance the overlap matrices $A_{m n}$ and $M_{m n}[2]$ ), or else read in from file (for instance the Kohn-Sham eigenvalues). These data are then passed from EPW to wannier90 through the wannier_run library routine. This feature of EPW ensures that the execution of wannier90 is embedded within EPW. Hence the end user is only required to run one single executable which communicates directly with wannier90. EPW also includes an option to pass additional input parameters to wannier90. This allows the user to access all the available features of wannier90, such as for instance plotting 
bandstructures or MLWFs. When called from EPW, wannier90 produces the gauge matrix $U_{m n}^{\mathbf{k}}[2]$, which yields the transformation between Bloch eigenstates and MWLFs, according to:

$$
w_{n \mathbf{R}_{e}}(\mathbf{r})=\frac{\Omega}{(2 \pi)^{3}} \int_{\mathrm{BZ}} d \mathbf{k} \mathrm{e}^{-i \mathbf{k} \cdot \mathbf{R}_{e}} \sum_{m} U_{m n}^{\mathbf{k}} \psi_{m \mathbf{k}}(\mathbf{r}),
$$

where $w_{n \mathbf{R}_{e}}$ is a MLWF associated with the direct lattice vector $\mathbf{R}_{e}, \Omega$ is the unit cell volume, and the integral is discretized over the Brillouin zone. The array $U_{m n}^{\mathbf{k}}$ has the dimensions of the number of Bloch bands times the number of MLWFs times the number of electronic wavevectors in the coarse Brillouin zone grid. This matrix is written to disk and can directly be read on subsequent program executions. The array $U_{m n}^{\mathbf{k}}$ is used in EPW in order to transform Bloch functions into MLWFs.

\section{Transformation from the Bloch representation on the coarse Brillouin zone grid to the MLWF representation (ephwann_shuffle)}

After calculating the electron-phonon matrix elements in the Bloch representation for each wavevector on the coarse electron and phonon Brillouin zone grids, EPW transforms the electronic Hamiltonian, the phonon dynamical matrix, and the electron-phonon matrix elements into the Wannier representation. Derivations and detailed explanations of the following transformations can be found in Ref. [15]. For clarity the electron band and the phonon mode indices will be omitted in the following equations. The electronic Hamiltonian in the MLWF representation $H_{\mathbf{R}_{e}, \mathbf{R}_{\mathrm{e}}^{\prime}}^{\mathrm{el}}$ is obtained as:

$$
H_{\mathbf{R}_{\mathrm{e}}, \mathbf{R}_{\mathrm{e}}^{\prime}}^{\mathrm{el}}=\sum_{\mathbf{k}} w_{\mathbf{k}} \mathrm{e}^{-i \mathbf{k} \cdot\left(\mathbf{R}_{\mathrm{e}}^{\prime}-\mathbf{R}_{\mathrm{e}}\right)} U_{\mathbf{k}}^{\dagger} H_{\mathbf{k}}^{\mathrm{el}} U_{\mathbf{k}}
$$

In this case the weights $w_{\mathbf{k}}$ are normalized to 1 . The Hamiltonian matrix elements in the Wannier representation $H_{\mathbf{R}_{\mathrm{e}}, \mathbf{R}_{\mathrm{e}}^{\prime}}^{\text {el }}$ decay rapidly with the distance $\left|\mathbf{R}_{\mathrm{e}}-\mathbf{R}_{\mathrm{e}}^{\prime}\right|$, as they scale with the overlap of MLWFs centered in the unit cells $\mathbf{R}=\mathbf{R}_{\mathrm{e}}$ and $\mathbf{R}=\mathbf{R}_{\mathrm{e}}^{\prime}$, respectively. The dynamical matrix can be transformed to a localized real-space representation using

$$
D_{\mathbf{R}_{\mathrm{p}}, \mathbf{R}_{\mathrm{p}}^{\prime}}^{\mathrm{ph}}=\sum_{\mathbf{q}} w_{\mathbf{q}} \mathrm{e}^{-i \mathbf{q} \cdot\left(\mathbf{R}_{\mathrm{p}}^{\prime}-\mathbf{R}_{\mathrm{p}}\right)} \mathbf{e}_{\mathbf{q}} D_{\mathbf{q}}^{\mathrm{ph}} \mathbf{e}_{\mathbf{q}}^{\dagger}
$$

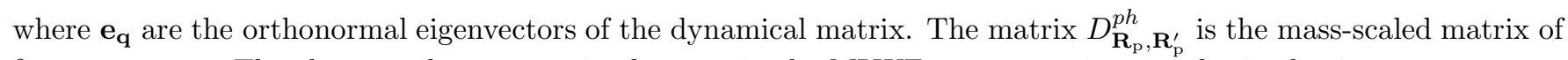
force constants. The electron-phonon matrix elements in the MLWF representation are obtained using:

$$
g\left(\mathbf{R}_{\mathrm{e}}, \mathbf{R}_{\mathrm{p}}\right)=\frac{1}{N_{\mathrm{p}}} \sum_{\mathbf{k}, \mathbf{q}} w_{\mathbf{k}} w_{\mathbf{q}} \mathrm{e}^{-i\left(\mathbf{k} \cdot \mathbf{R}_{\mathrm{e}}+\mathbf{q} \cdot \mathbf{R}_{\mathrm{p}}\right)} U_{\mathbf{k}+\mathbf{q}}^{\dagger} g(\mathbf{k}, \mathbf{q}) U_{\mathbf{k}} \mathbf{u}_{\mathbf{q}}^{-1}
$$

where the $\mathbf{u}_{\mathbf{q} \kappa}^{\nu}$ are the phonon eigenvectors scaled by the atomic masses [15]. In order to check the spatial decay of $H_{\mathbf{R}_{\mathrm{e}}, 0}^{\mathrm{el}}, D_{\mathbf{R}_{\mathrm{p}}, 0}^{\mathrm{ph}}$, and $g\left(\mathbf{R}_{\mathrm{e}}, \mathbf{R}_{\mathrm{p}}\right)$ the magnitude of these quantites as a function of $\mathbf{R}_{\mathrm{e}}$ and $\mathbf{R}_{\mathrm{p}}$ is written to formatted files in the working directory. A run-time option allows for all data in the Wannier representation to be written into one single file. For subsequent calculations these data can be read in and program execution can restart without the need to go through the prior computational steps.

\section{E. Tranformation from the MLWF representation to the Bloch representation on the fine Brilluin zone grid (ephwann_shuffle)}

The accuracy of EPW calculations depends on the spatial localization of the MLWFs and the phonon perturbations. Typically MLWFs are localized within a few $\AA[39,40]$. The localization of the phonon perturbation is dependent upon the dielectric screening properties of the system, and must be verified in each case before proceeding with the interpolation [15].

In order to calculate the electronic eigenstates, the phonon frequencies, and the electron-phonon matrix elements on a fine Brillouin zone grid, the Hamiltonian, the dynamical matrix, and the electron-phonon matrix elements are truncated outside of a real-space supercell containing $N_{\mathbf{k}}$ and $N_{\mathbf{q}}$ unit cells in the case of electrons and phonons, 
respectively. Here $N_{\mathbf{k}}$ and $N_{\mathbf{q}}$ are the number of grid points in the coarse Brillouin zone meshes. Following this truncation it is possible to perform an interpolation back into the Bloch representation onto arbitrary electron and phonon wavevectors. The Hamiltonian, the dynamical matrix, and the electron-phonon matrix elements are Fouriertransformed back to the Bloch representation by inverting Eqs. (11), (12), and (13) [15].

The procedure described here enables the calculation of electron-phonon matrix elements on extremely fine Brillouin zone grids. The fine grids of electron and phonon wavevectors are specified by the user in input. The procedure adopted here is similar to the Fourier interpolation of time series commonly adopted in signal processing [41]. The same strategy as the one employed in EPW has been adopted in order to study Fermi surfaces [18] and the anomalous Hall effect [20].

At this stage the Hamiltonian, the dynamical matrix, and the electron-phonon matrix elements on fine grids of electron and phonon wavevectors are used to compute the physical quantities described in Sec. IIIA

\section{F. Summary of the tasks executed by EPW}

The computational steps described in the previous sections can be summarized schematically as follows:

1. Electron eigenstates and eigenvalues are read from disk,

2. wannier90 input data including the overlap matrices $A_{m n}$ and $M_{m n}$ are computed,

3. wannier90 is called as a library, and the resulting MLWF localization matrix is stored on disk.

4. The electron-phonon matrix elements are computed on a coarse grids of electron and phonon wavevectors in the Bloch representation,

5. The Hamiltonian, dynamical matrix, and electron-phonon matrix elements are transformed from the Bloch representation to the MLWF representation,

6. The Hamiltonian, dynamical matrix, and electron-phonon matrix elements are Fourier-transformed back to the Bloch representation on arbitrarily dense grids of electron and phonon wavevectors,

7. The electronic eigenvalues, phonon frequencies, and electron-phonon matrix elements are processed in order to calculate physical quantites such as, for instance, the electron self-energy or the electron-phonon coupling strength.

\section{PARALlELIZATION}

EPW is designed and optimized to be executed on multiple processors. Within the Quantum-ESPRESSO package, parallel tasks are split into processor "pools", where each pool is allocated a set of electronic wavevectors k. If there is more than one processor within each pool, the reciprocal space $\mathbf{G}$-vectors for describing the wavefunctions are split amongst the processors of that pool. EPW can be executed in parallel by splitting the calculation over electron or phonon wavevectors. EPW is not parallelized over the reciprocal-space G-vectors. The parallelization strategy in EPW is tailored to each step of the computation. As execution of EPW begins, the coarse k-point mesh is generated and distributed amongst the available pools, as in Quantum-ESPRESSO.

Most of the inputs to the wannier90 such as the lattice and reciprocal vectors are passed through Quantum-ESPRESSO and EPW. Important exceptions are the overlap matrices $A_{m n}$ and $M_{m n}$. Within EPW the computation of the overlap matrix elements is distributed across the coarse grid k-point pools. As the elements of the matrices $A_{m n}$ and $M_{m n}$ are computed independently, the execution time of this step scales inversely with the the number of pools.

wannier90 is executed serially on each processor through a library call. The time required to determine MLWFs is negligible as compared to the computation of the electron-phonon matrix elements on the coarse Brillouin zone grids.

The electron-phonon matrix elements on the coarse Brillouin zone grid are computed sequentially for each phonon wavevector, while the electron wavevectors are distributed across pools.

The computation of the electron-phonon matrix elements on the fine Brillouin zone grids is parallelized over electronic or phonon wavevectors depending on the calculation type. For example, when calculating the phonon self-energy an integration is required over electronic wavevectors. In this case the most convenient strategy is to parallelize over the electronic wavevectors. The converse is true for the calculation of the electron self-energy. In those cases where the final quantity requires integrations over both electron and phonon wavevectors, such as the total electron-phonon coupling strength, the efficiency of EPW is independent of the choice of the parallelization scheme. 

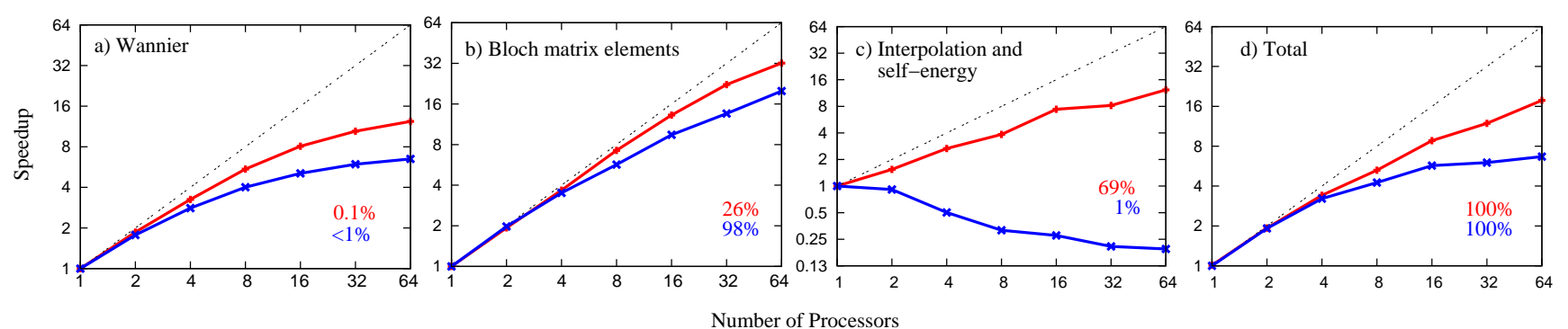

FIG. 1: Parallelization of EPW: speedup vs number of processors observed in parallelizing a) the generation of MLWFs, b) the calculation of the electron-phonon matrix elements on the coarse Brillouin zone grids, c) the interpolation of the electron-phonon matrix elements to the fine Brillouin zone grids as well as the computation of the phonon self-energy. Panel d) displays the speedup observed to perform the entire computation including the initialization step and the intermediate I/O. The speedup is defined as the ratio between the time it takes to run a calculation on a single processor and the time it takes to run the same calculation on a given number of processors. The diagonal dotted lines correspond to the ideal speedup, which is equal to the number of processors employed. The calculations have been performed for hole-doped $\mathrm{SiC}$ in the rigid-band approximation. The unit cell contains 2 atoms. The blue line corresponds to a calculation with coarse Brillouin zone grids containing $6 \times 6 \times 6$ k- and q-points, a kinetic energy cutoff of $30 \mathrm{Ry}$, and fine Brillouin zone grids containing 1000 points each. The red line corresponds to a calculation with coarse Brillouin zone grids containing $8 \times 8 \times 8 \mathbf{k}$ - and $\mathbf{q}$-points, a kinetic energy cutoff of 60 Ry, and fine $\mathbf{k}$ - and q-points grids containing $10^{6}$ and 104 points (the irreducible points of a uniform and unshifted $14 \times 14 \times 14$ grid), respectively. The parallelization becomes more efficient as the number of electron and phonon wavevectors in the fine Brillouin zone grids is increased.

Figure 1 shows the efficiency of our parallelization strategy for various sections of EPW for the test case of hole-doped $\mathrm{SiC}$. This example is included for reference in the EPW distribution.

\section{A. Calculations "on the fly"}

The interpolation part of EPW can be executed in two different modes. In the first mode EPW calculates all the electron-phonon matrix elements for every $\mathbf{k}$ - and q-point of the fine Brillouin zone grids, and stores this information to disk. The subroutines for calculating the electron or phonon self-energy are then called and the matrix elements along with the electronic eigenvalues and the phonon frequencies are read from disk in order to evaluate Eqs. (11), (9).

In the second mode of operation, the electron or the phonon self-energy are calculated "on the fly" as the matrix elements are generated, and all the quantities are overwritten. In this case the matrix elements are not stored on disk.

This feature allows us to address systems for which the storage required for the electron-phonon matrix elements would be exceedingly large. For example, if the fine electron and phonon Brillouin zone grids consisted each of $10^{5}$ wavevectors, and we had 8 MLWFs and 6 phonon modes, then the entire set of double precision matrix elements would require more than $1 \mathrm{~TB}$ of storage. On the other hand the calculation on the fly would reduce the storage requirement down to a manageable size of $1 \mathrm{~TB}$.

\section{EXAMPLES}

In the following we illustrate the capabilities of EPW by describing three prototypical systems: (i) Lead, a simple metal which is also a strong-coupling superconductor. (ii) Graphene, a two-dimensional zero-gap semiconductor with linear quasiparticle dispersions close to the Fermi level. (iii) Silicon carbide, a wide-gap semiconductor which becomes a low-temperature superconductor upon boron doping.

\section{A. Lead}

Solid $\mathrm{Pb}$ is the prototypical strong-coupling superconductor. While the superconducting transition temperature of $\mathrm{Pb}$ is less than $10 \mathrm{~K}$, the electron-phonon coupling strength has been measured and calculated to be very large, in the range of $\lambda=1.3 \pm 0.1$ [42 46]. 

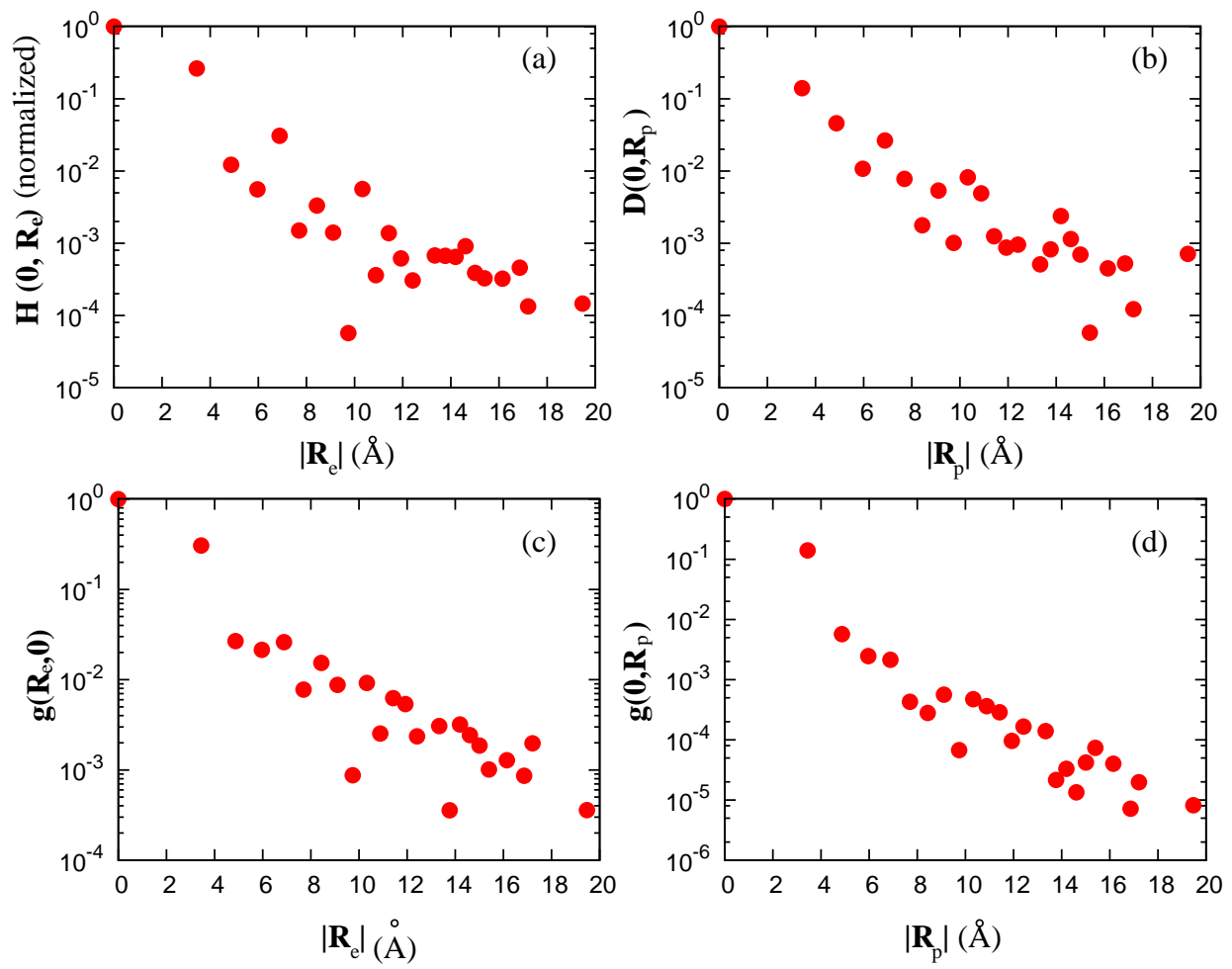

FIG. 2: Spatial decay of the largest components of the Hamiltonian $H_{\mathbf{R}_{\mathrm{e}}, 0}^{\mathrm{el}}$ (a), the dynamical matrix $D_{\mathbf{R}_{\mathrm{p}}, 0}^{\mathrm{ph}}(\mathrm{b})$, and the electron-phonon matrix elements $g\left(0, \mathbf{R}_{\mathrm{p}}\right)$ (c) and $g\left(\mathbf{R}_{\mathrm{e}}, 0\right)$ (d) for fcc $\mathrm{Pb}$. The data are plotted as a function of distance and are taken along several directions.

In this section we present an example calculation of the electron-phonon coupling in bulk $\mathrm{Pb}$ and we compare our results to experimental data. The calculations were performed within the local-density approximation to density functional theory using Quantum-ESPRESSO, wannier90, and EPW. A norm-conserving, scalar-relativistic pseudopotential including four valence electrons was used to take into account the core-valence interaction. The electronic states were computed within a plane-wave basis with a kinetic energy cutoff of $80 \mathrm{Ry}$. The charge density was computed self-consisently on a $16 \times 16 \times 16 \Gamma$-centered Brillouin zone grid. The electronic wavefunctions used within EPW were calculated on an $8 \times 8 \times 8$ uniform Brillouin zone grid. The phonon dynamical matrices and the linear variations of the self-consistent potential were calculated within DFPT using the Quantum-ESPRESSO package, using the same convergence parameters as above. We considered a uniform $8 \times 8 \times 8$ Brillouin zone grid for the phonon calculations, corresponding to 29 irreducible phonon wavevectors.

Four Wannier states were used to reconstruct the electronic structure near the Fermi level. These states were found to be $s p^{3}$-like functions localized symmetrically along each of the $\mathrm{Pb}-\mathrm{Pb}$ bonds, with a spatial spread of $2.17 \AA$. The spatial decay of the electron Hamiltonian, the phonon dynamical matrix, and the electron-phonon matrix elements in the MLWF representation are shown in Fig. 2.

In order to calculate the phonon linewidths and the total electron-phonon coupling, the electron Hamiltonian, the phonon dynamical matrix, and the electron-phonon matrix elements were transformed from the MLWF representation to the Bloch representation by the generalized Fourier interpolation described in Sec. IIIE, By using $10^{6}$ k-points, 8000 q-points, and a smearing parameter of $30 \mathrm{meV}$, we obtained a total electron-phonon coupling strength $\lambda=1.41$. The Eliashberg spectral function $\alpha^{2} F(\omega)$ is shown in Fig. 3. together with the experimental curve obtained by inverting tunneling data [42]. Our method clearly yields a very good agreement with experiment.

For comparison with experiment we also calculated the superconducting transition temperature $T_{\mathrm{c}}$ starting from the total electron-phonon coupling. We adopted the modified McMillan equation [47] and a Coulomb repulsion parameter $\mu^{*}$ between 0.10 and 0.15 , obtaining $T_{c}$ equal to $4 \mathrm{~K}$ to $6 \mathrm{~K}$ respectively. This result compares favorably to the experimental value $7.2 \mathrm{~K}$. 


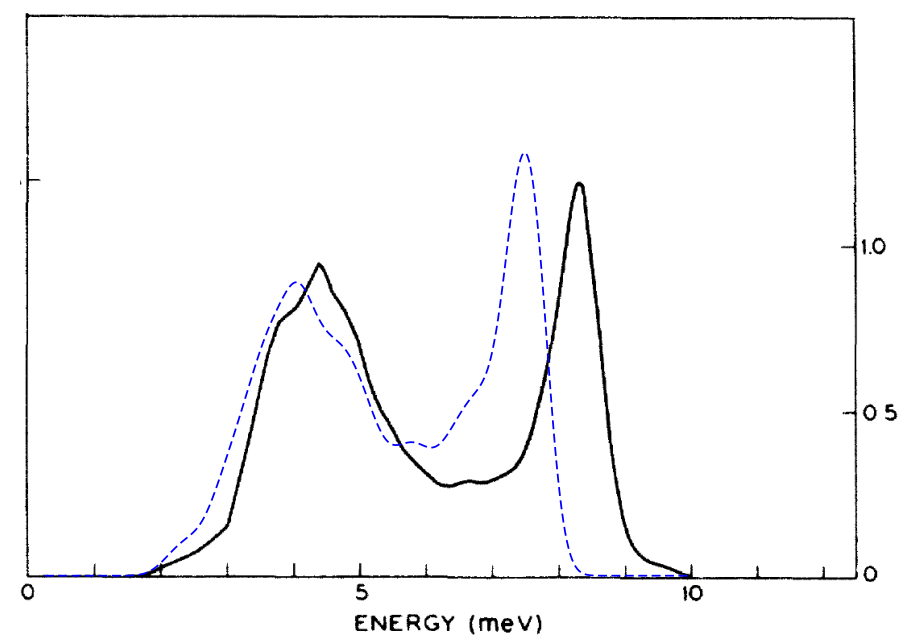

FIG. 3: Eliashberg spectral function of fcc Pb. The solid line is from Ref. [42], the dashed line has been obtained using EPW.

\section{B. Graphene}

Graphene is a two-dimensional sheet of carbon atoms which has attracted enourmous attention from the scientific community due to its peculiar electronic properties [48], and in particular the Dirac-like nature of electrons near the Fermi level [49, 50]. Considerable attention has been paid to the measurement of many-body renormalization effects, such as the effect of the electron-phonon interaction on the electronic bandstructure, using for instance angle-resolved photoemission experiments [51].

We here present the results of an electron-phonon calculation on a sheet of freestanding graphene [23]. The local density approximation to density functional theory was employed in conjunction with a norm-conserving carbon pseudopotential. A plane wave basis with a kinetic energy cutoff of 60 Ry was used. The graphene sheets were separated by a vacuum of $8 \AA$ in a supercell geometry in order to eliminate spurious interactions between periodic replicas. The relaxed C-C bond length was found to be $1.405 \AA$.

Three in-plane bonding Wannier functions, two $p_{z}$-like Wannier functions (one per each carbon atom), and two $s$-like Wannier functions directly above and below the center of the hexagon (away from the graphene plane by 1.57 $\AA$ ) in the two-atom unit cell were used to describe the electronic structure. The spatial spread of these MLWFs are $0.565 \AA, 0.782 \AA$, and $1.726 \AA$, respectively. The spatial decay of the electron Hamiltonian, the phonon dynamical matrix, and the electron-phonon matrix elements in the MLWF representation for a $6 \times 6 \times 1$ grid are shown in Fig. 4.

The electron self-energy was computed and the integral of of Eq. (9) was performed with $10^{6}$ phonon q-vectors, obtained through the interpolation method of this communication. The mass renormalization parameter, $\lambda_{n \mathbf{k}}$ has been obtained as an energy derivative of the electron self-energy as in $\lambda_{n \mathbf{k}}=\left.\frac{\partial}{\partial \epsilon} \operatorname{Re} \Sigma_{n \mathbf{k}}\left(\epsilon_{n \mathbf{k}}\right)\right|_{\epsilon=\epsilon_{F}}$. The results of the calculations of the real and imaginary parts of the electron self-energy as well as the electron-phonon coupling parameter as a function of Fermi energy are given in Fig. 5 .

\section{Silicon carbide}

The possibility of achieving superconducting behavior in semiconductors has recently attracted considerable interest, and several experimental [52, 53] and theoretical [22, 27, 54 59] studies have been performed on this class of materials. Boron-doped $\mathrm{SiC}$ is a promising candidate because of its potential uses in power electronics owing to its large breakdown voltage.

For this test case we performed electron-phonon calculations on a rigid-band model of $4 \%$ hole-doped cubic SiC. For our calculations we employed the local density approximation to density functional theory, norm-conserving pseudopotentials, and a plane wave basis with a kinetic energy cutoff of 60 Ry. The relaxed lattice parameter was found to be $4.34 \AA$, in good agreement with the experimental value of $4.36 \AA$. EPW was executed using coarse meshes of $8 \times 8 \times 8$ uniform grids of electron and phonon wavevectors. Four Wannier functions per formula unit were considered to describe the valence electronic struture. MLWFs were found to be $s p_{3}$-like functions with an average spread of 

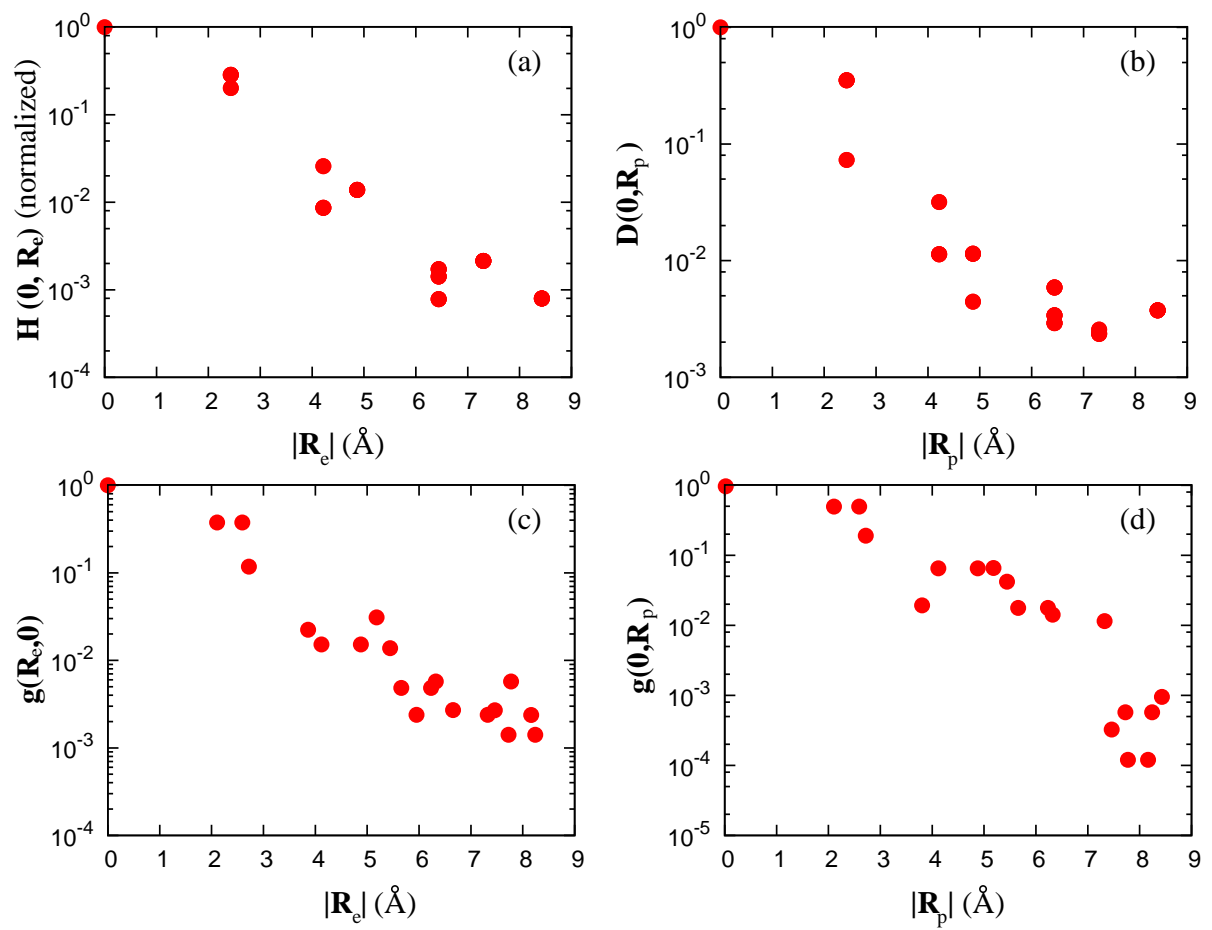

FIG. 4: Spatial decay of the largest components of the Hamiltonian $H_{\mathbf{R}_{\mathrm{e}}, 0}^{\mathrm{el}}$ (a), the dynamical matrix $D_{\mathbf{R}_{\mathrm{p}}, 0}^{\mathrm{ph}}$ (b), and the electron-phonon matrix elements $g\left(0, \mathbf{R}_{\mathrm{p}}\right)(\mathrm{c})$ and $g\left(\mathbf{R}_{\mathrm{e}}, 0\right)(\mathrm{d})$ for graphene. The data are plotted as a function of distance and are taken along several directions.
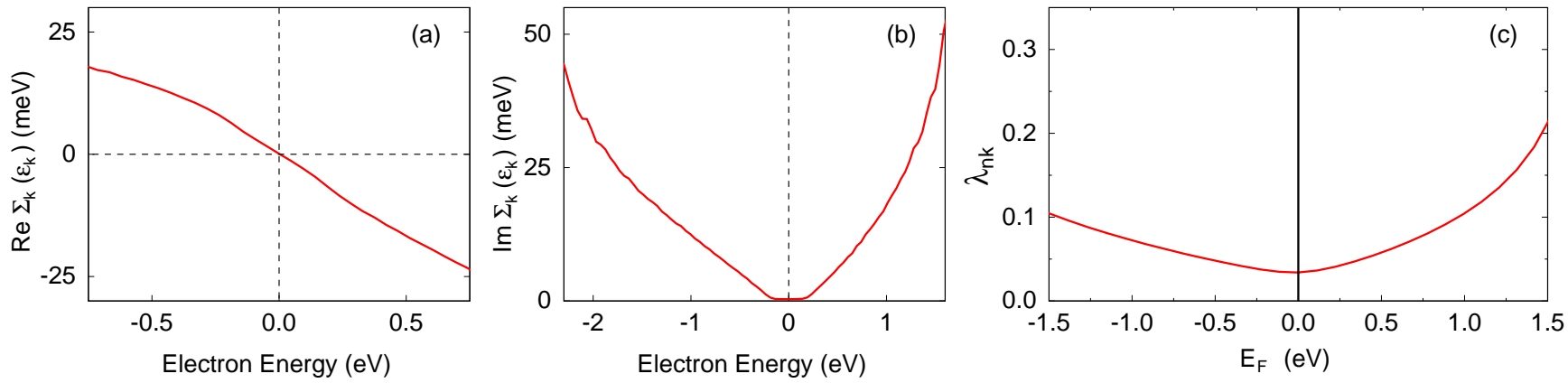

FIG. 5: The real (a) and imaginary (b) parts of the electron self-energy $\Sigma_{\mathbf{k}}$ near the Dirac point as well as the electron-phonon coupling $\lambda_{n \mathbf{k}}$ as a function of doping (c) for graphene. In panels (a) and (b), the Fermi energy has been set to zero.

$1.15 \AA$. The spatial decay of the electron Hamiltonian, the phonon dynamical matrix, and the electron-phonon matrix elements in the MLWF representation are shown in Fig. 6. We calculated the total electron-phonon coupling strength $\lambda=0.34$ using 250,000 k-points and 8000 q-points. The superconducting transition temperature was calculated using a Coulomb repulsion parameter $\mu^{\star}=0.1$ and was found to be $T_{\mathrm{c}}=1 \mathrm{~K}$. Our result is in good agreement with recent experimental data yielding $T_{\mathrm{c}}=1.4 \mathrm{~K}[52]$.

\section{CONCLUSION}

In this communication we introduced EPW, a computer code for calculating the electron-phonon coupling from first-principles using density functional perturbation theory and maximally localized Wannier functions. EPW enables extremely accurate and highly efficient calculations of the mode-resolved and total electron-phonon coupling strength, as well as phonon and electron linewidths. The code is distributed through the website http://epw.org.uk and is 

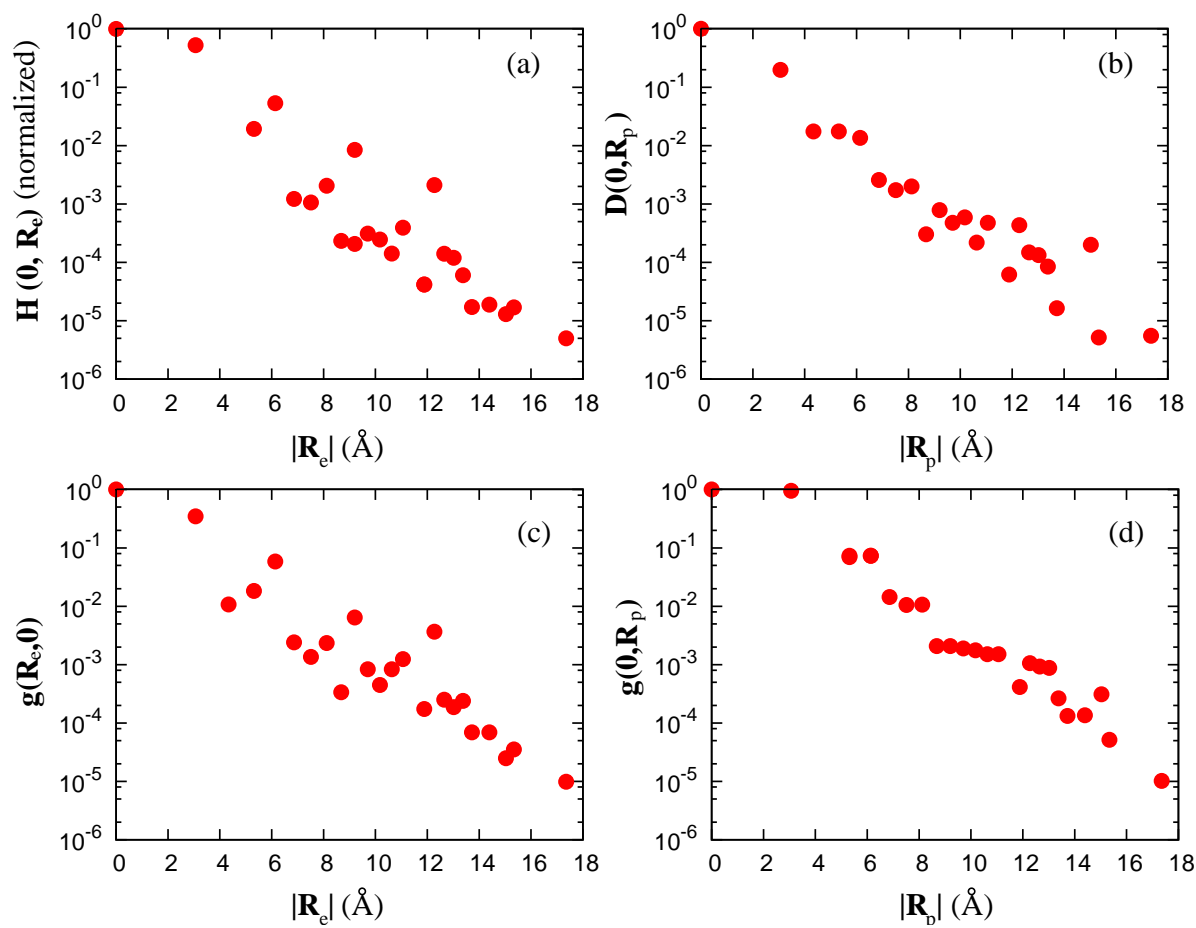

FIG. 6: Spatial decay of the largest components of the Hamiltonian $H_{\mathbf{R}_{\mathrm{e}}, 0}^{\mathrm{el}}$ (a), the dynamical matrix $D_{\mathbf{R}_{\mathrm{p}}, 0}^{\mathrm{ph}}$ (b), and the electron-phonon matrix elements $g\left(0, \mathbf{R}_{\mathrm{p}}\right)(\mathrm{c})$ and $g\left(\mathbf{R}_{\mathrm{e}}, 0\right)$ (d) for cubic hole-doped SiC. The data are plotted as a function of distance and are taken along several directions.

available under the terms of the GNU General Public License. Plans are in place to extend EPW in order to implement the anisotropic Eliashberg theory [60], the density functional theory for superconductors [61, 62], and phonon-assisted optical responses [63].

\section{APPENDIX}

\section{A. Specifying a unique gauge for the electronic wavefunctions (setphases)}

Since nondegenerate electronic wavefunctions are uniquely defined up to a phase, and a set of degenerate wavefunctions can be mixed via a unitary matrix, electron-phonon matrix elements are machine dependent. In some cases it may be convenient to use electron-phonon matrix elements outside of EPW, for instance in the study of phonon sidebands in excitonic spectra [64], or in the phonon-assisted Auger recombination [65]. In order to meaningfully use the electron-phonon matrix elements generated by EPW outside of the code it is necessary to define uniquely the phase of each wavefunction as well as the way in which degenerate wavefunctions are mixed.

In order to set the gauge of each wavefunction in EPW we proceed as follows. First, we determine the subset of degenerate wavefunctions at each wavevector on the coarse mesh. Then we artificially lift the degeneracies by diagonalizing the subset of degenerate states with respect to a small external perturbation. In principle the perturbation could take on any form, however for convenience EPW employs a nonlinear combination of the data found in the prefix.dvscf files. Finally, the Fourier components of each wavefunction are scaled by a complex factor $\exp (i \theta)$ in such a way that the largest component of each wavefunction is real-valued. In this way, the machine dependence of the matrix elements is eliminated. This procedure is explained in detail in Ref. [15].

\section{ACKNOWLEDGMENTS}

We are grateful to Arash A. Mostofi, Jonathan R. Yates, Ivo Souza, David Vanderbilt, and Nicola Marzari for useful interactions about wannier90, Stefano de Gironcoli and Paolo Giannozzi for interactions on Quantum-ESPRESSO, and 
John M. Rowell for permission to use the figure contained in Fig. 3. J.N. and development of EPW was supported by National Science Foundation Grant No. DMR07-05941. B.D.M. and codes testing was supported by the Director, Office of Science, Office of Basic Energy Sciences, Materials Sciences and Engineering Division, U.S. Department of Energy under Contract No. DE-AC02-05CH11231. C.-H.P. was supported by Office of Naval Research MURI Grant No. N00014-09-1066. The research leading to these results has received funding from the European Research Council under the European Community's Seventh Framework Programme (FP7/2007-2013) / ERC grant agreement no. 239578.

* Electronic address: jnoffsinger@civet.berkeley.edu

[1] Giannozzi, P. et al., Journal of Physics: Condensed Matter 21 (2009).

[2] Mostofi, A. A. et al., Computer Physics Communications 178 (2008) 685 .

[3] Mott, N. F., Proc. Phys. Soc. London 46 (1934), and references therein.

[4] Schrieffer, J. R., Theory of Superconductivity, Benjamin, New York, 1964.

[5] MacDonald, D. K. C., Thermoelectricity: An Introduction to the Principles, Wiley, New York, 1962.

[6] Kohn, W., Phys. Rev. Lett. 2 (1959).

[7] Peierls, R. E., Quantum Theory of Solids, Clarendon, Oxford, 1964.

[8] Damascelli, A., Hussain, Z., and Shen, Z.-X., Rev. Mod. Phys. 75 (2003) 473.

[9] Pintschovius, L., Physica Status Solidi B 242 (2004) 30.

[10] Cardona, M., and Thewalt, M. L. W., Rev. Mod. Phys. 77 (2005) 1173.

[11] Lam, P. K., Dacorogna, M. M., and Cohen, M. L., Phys. Rev. B 34 (1986) 5065.

[12] Ihm, J., Zunger, A., and Cohen, M. L., Journal of Physics C: Solid State Physics 12 (1979) 4409.

[13] Ceperley, D. M. and Alder, B. J., Phys. Rev. Lett. 45 (1980) 566.

[14] Baroni, S., de Gironcoli, S., and Corso, A. D., Rev. Mod. Phys. 73 (2001) 515.

[15] Giustino, F., Cohen, M. L., and Louie, S. G., Physical Review B 76 (2007) 165108.

[16] Marzari, N. and Vanderbilt, D., Phys. Rev. B 56 (1997) 12847.

[17] Souza, I., Marzari, N., and Vanderbilt, D., Phys. Rev. B 65 (2001) 035109.

[18] Yates, J. R., Wang, X., Vanderbilt, D., and Souza, I., Phys. Rev. B 75 (2007) 195121.

[19] Wang, X., Vanderbilt, D., Yates, J. R., and Souza, I., Phys. Rev. B 76 (2007) 195109.

[20] Wang, X., Yates, J. R., Souza, I., and Vanderbilt, D., Phys. Rev. B 74 (2006) 195118.

[21] Hamann, D. R. and Vanderbilt, D., Phys. Rev. B 79 (2009) 045109.

[22] Giustino, F., Yates, J. R., Souza, I., Cohen, M. L., and Louie, S. G., Phys. Rev. Lett. 98 (2007) 047005.

[23] Park, C.-H., Giustino, F., Cohen, M. L., and Louie, S. G., Phys. Rev. Lett. 99 (2007) 086804.

[24] Park, C.-H. et al., Phys. Rev. B 77 (2008) 113410.

[25] Noffsinger, J., Giustino, F., Louie, S. G., and Cohen, M. L., Phys. Rev. B 77 (2008) 180507.

[26] Park, C.-H., Giustino, F., Spataru, C. D., Cohen, M. L., and Louie, S. G., Phys. Rev. Lett. 102 (2009) 076803.

[27] Noffsinger, J., Giustino, F., Louie, S. G., and Cohen, M. L., Phys. Rev. B 79 (2009) 104511.

[28] Giustino, F., Cohen, M. L., and Louie, S. G., Nature (London) 452 (2008) 975.

[29] Park, C.-H., Giustino, F., Cohen, M. L., and Louie, S. G., Nano Lett. 8 (2008) 4229.

[30] Park, C.-H., Giustino, F., Spataru, C. D., Cohen, M. L., and Louie, S. G., Nano Lett. 9 (2009) 4234.

[31] Fuchs, M. and Scheffler, M., Computer Physics Communications 119 (1999) 67.

[32] Cohen, M. L., Physica Scripta 5 (1982).

[33] Troullier, N. and Martins, J. L., Phys. Rev. B 43 (1991) 1993.

[34] Perdew, J. P. and Zunger, A., Phys. Rev. B 23 (1981) 5048.

[35] Vanderbilt, D., Phys. Rev. B 41 (1990) 7892.

[36] Allen, P. B. and Mikovic, B., in Solid State Physics, edited by Ehrenreich, H., Seitz, F., and Turnbull, D., Volume 37, page 1, Academic, New York, 1982.

[37] Migdal, A. B., Zh. Eksp. Teor. Fiz. 34 (1958), [Sov. Phys. JETP 7, 996 (1958)].

[38] Savrasov, S. Y. and Savrasov, D. Y., Phys. Rev. B 54 (1996) 16487.

[39] Cloizeaux, J. D., Phys. Rev. 135 (1964) A698.

[40] Nenciu, G., Commun. Math. Phys. 91 (1983) 81.

[41] Unser, M., Proceedings of the IEEE 88 (2000) 569.

[42] McMillan, W. L. and Rowell, J. M., Phys. Rev. Lett. 14 (1965) 108.

[43] Allen, P. B. and Cohen, M. L., Phys. Rev. 187 (1969) 525.

[44] Knorr, R. and Barth, N., J. Low Temp. Phys. 4 (1971) 469.

[45] Franck, J. P., Keeler, W. J., and Wu, T. M., Solid State Communications 7 (1969) 483.

[46] Noffsinger, J. and Cohen, M. L., to be published.

[47] Allen, P. B. and Dynes, R. C., Phys. Rev. B 12 (1975) 905.

[48] Novoselov, K. S. et al., Proc. Natl. Acad. Sci. U.S.A. 102 (2005) 451.

[49] Novoselov, K. S. et al., Nature (London) 438 (2005) 7065. 
[50] Zhang, Y., Tan, Y.-W., Stormer, H. L., and Kim, P., Nature (London) 438 (2005) 7065.

[51] Bostwick, A., Ohta, T., Seyller, T., Horn, K., and Rotenberg, E., Nature Phys. 3 (2007) 36.

[52] Ren, Z.-A. et al., J. Phys. Soc. Jpn. 76 (2007) 103710.

[53] Ekimov, E. A. et al., Nature (London) 428 (2004) 542.

[54] Margine, E. R. and Blase, X., Appl. Phys. Lett. 93 (2008) 192510.

[55] Cohen, M. L., Rev. Mod. Phys. 36 (1964) 240.

[56] Lee, K.-W. and Pickett, W. E., Phys. Rev. Lett. 93 (2004) 237003.

[57] Boeri, L., Kortus, J., and Andersen, O. K., Phys. Rev. Lett. 93 (2004) 237002.

[58] Ma, Y. et al., Phys. Rev. B 72 (2005) 014306.

[59] Blase, X., Adessi, C., and Connétable, D., Phys. Rev. Lett. 93 (2004) 237004.

[60] Choi, H. J., Roundy, D., Sun, H., Cohen, M. L., and Louie, S. G., Nature (London) 418 (2002) 758.

[61] Oliveira, L. N., Gross, E. K. U., and Kohn, W., Phys. Rev. Lett. 60 (1988) 2430.

[62] Wacker, O. J., Kümmel, R., and Gross, E. K. U., Phys. Rev. Lett. 73 (1994) 2915.

[63] Beattie, A. R. and Landsberg, P. T., Proc. R. Soc. London, Ser. A 249 (1959).

[64] Perebeinos, V., Tersoff, J., and Avouris, P., Phys. Rev. Lett. 94 (2005) 027402.

[65] Lochmann, W. and Haug, A., Solid State Communications 35 (1980) 553. 